\title{
Provider- and patient-level predictors of oral anticancer agent initiation and adherence in patients with metastatic renal cell carcinoma
}

\author{
Lisa P. Spees $^{1,2}$ () | Stephanie B. Wheeler ${ }^{1,2}$ | Bradford E. Jackson ${ }^{2}$ | \\ Christopher D. Baggett ${ }^{2,3}$ | Lauren E. Wilson ${ }^{4}$ | Melissa A. Greiner ${ }^{4}$ | Deborah R. Kaye ${ }^{5,6}$ \\ Tian Zhang ${ }^{5,6,7}$ | $\mid$ Daniel George ${ }^{5,6}$ | Charles D. Scales Jr ${ }^{4,7}$ | Jessica E. Pritchard ${ }^{4}$ | \\ Michael Leapman $^{8,9}$ | Cary P. Gross ${ }^{9,10}$ @ | Michaela A. Dinan ${ }^{9,11}$
}

${ }^{1}$ Department of Health Policy and Management, Gillings School of Global Public Health, University of North Carolina at Chapel Hill (UNC-CH), Chapel Hill, North Carolina, USA

${ }^{2}$ Lineberger Comprehensive Cancer Center (LCCC), UNC-CH, Chapel Hill, North Carolina, USA

${ }^{3}$ Department of Epidemiology, Gillings School of Global Public Health, UNC$\mathrm{CH}$, Chapel Hill, North Carolina, USA

${ }^{4}$ Department of Population Health Sciences, Duke University School of Medicine (DUSM), Durham, North Carolina, USA

${ }^{5}$ Duke Cancer Institute (DCI) Center for Prostate and Urologic Cancers, Durham, North Carolina, USA

${ }^{6}$ Department of Medicine, DUSM, Durham, North Carolina, USA

${ }^{7}$ Department of Surgery (Urology), DUSM, Durham, North Carolina, USA

${ }^{8}$ Department of Urology, Yale School of Medicine, New Haven, Connecticut, USA

${ }^{9}$ Cancer Outcomes, Public Policy, and Effectiveness Research (COPPER) Center, Yale School of Medicine, New Haven, Connecticut, USA

${ }^{10}$ Department of Medicine, Yale School of Medicine, New Haven, Connecticut, USA

\begin{abstract}
Background: Improving oral anticancer agent (OAA) initiation and adherence is the important quality-of-care issues, particularly since one fourth of anticancer agents being developed will be administered orally. Our objective was to identify provider- and patient-level characteristics associated with OAA initiation and adherence among individuals with metastatic renal cell carcinoma (mRCC).

Methods: We used state cancer registry data linked to multi-payer claims data to identify patients with mRCC diagnosed in 2004-2015. Provider data were obtained from North Carolina Health Professions Data System and the National Plan \& Provider Enumeration System. We estimated risk ratios (RRs) and corresponding 95\% confidence limits (CLs) using modified Poisson regression to evaluate factors associated with OAA initiation and adherence.

Results: Among the 207 (out of 687) patients who initiated an OAA following mRCC diagnosis and survived 90 days, median proportion of days covered was 0.91. Patients with a modal provider specializing in hematology/medical oncology were much more likely to initiate OAAs than those seen by other specialties. Additionally, patients with a female provider were more likely to initiate OAAs than those with a male provider. Compared to patients treated by providers practicing in both urban and rural areas, patients with providers practicing solely in urban areas were more likely to initiate OAAs, after controlling for patient-level factors ( $\mathrm{RR}=1.37$; 95\% CL: 1.09-1.73). Medicare patients were less likely to be adherent than those with private insurance ( $R R=0.61 ; 95 \%$ CL: $0.42-0.87)$.

Conclusions: Our results suggest that provider- and patient-level factors influence OAA initiation in patients with mRCC but only insurance type was associated with adherence.
\end{abstract}


${ }^{11}$ Department of Chronic Disease Epidemiology, Yale School of Public Health, New Haven, Connecticut, USA

\section{Correspondence}

Lisa P. Spees, Department of Health Policy and Management, Gillings School of Global Public Health, University of North Carolina at Chapel Hill (UNC-CH), Chapel Hill, NC 27599, USA.

Email: 1spees21@live.unc.edu

\section{Funding information}

Research reported in this publication was supported by the National Cancer Institute of the National Institutes of Health (NIH) (R01CA226842). Federal money is financing $100 \%$ of the cost. Database infrastructure was supported through the UNC Clinical and Translational Science Award (UL1TR001111) and the UNC LCCC, University Cancer Research Fund via the State of North Carolina. The content is solely the responsibility of the authors and does not necessarily represent the official views of the NIH.

\section{K E Y W O R D S}

adherence, initiation, metastatic, oral anticancer agents, renal cell carcinoma

\section{1 | INTRODUCTION}

Renal cell carcinoma (RCC) leads to more years of life lost than any other genitourinary cancer in the United States. ${ }^{1,2}$ An estimated 73,750 new cases of RCC are diagnosed annually, and approximately 558,000 patients currently live with the disease. Although survival is excellent for patients with localized RCC, only $13 \%$ of those diagnosed with metastatic disease will survive 5 years. ${ }^{2}$ Since 2005, the US Food and Drug Administration (FDA) have approved several first- and second-line oral anticancer agents (OAAs) to treat metastatic renal cell carcinoma (mRCC). ${ }^{3}$ These include first- and second-generation tyrosine kinase inhibitors whose pharmacokinetics require oral administration and offer convenience relative to infused therapies. Given that one fourth of anticancer agents being developed will be administered orally, ${ }^{4,5}$ improving OAA initiation and adherence has become an important quality-of-care issue.

Despite increased use, few published studies have examined the predictors of OAA initiation and adherence among mRCC patients in real-world settings. ${ }^{6-10}$ While several patient-level factors such as socioeconomic status, race/ethnicity, age, and comorbidities have been associated with OAA initiation and adherence for mRCC and other cancers, few provider-level factors have not been examined. Providers not only control access to OAAs but also influence OAA adherence; previous work suggests that providers' knowledge of OAAs as well as their attitudes and support of OAAs influence adherence. ${ }^{11,12}$ To optimize the outcomes in mRCC patients, multiple levels of influence within the healthcare system, including provider factors, need to be taken into account, and a better understanding of the larger context in which medicationtaking barriers should be addressed is needed. ${ }^{13-15}$ Our study examined both provider- and patient-level factors associated with initiation of and adherence to OAAs in a real-world cohort of individuals newly diagnosed with mRCC.

\section{2 | METHODS}

\section{1 | Study population}

We conducted a retrospective cohort study of patients diagnosed with mRCC and their providers between 2004 and 2015. Patient data were obtained from the University of North Carolina Cancer Information Population Health Resource, a resource that links the North Carolina Central Cancer Registry data to administrative claims data from private health insurance, Medicare, and Medicaid plans 
across the state. ${ }^{16}$ Provider data were obtained from North Carolina Health Professions Data System and the National Plan \& Provider Enumeration System.

Figure 1 illustrates how we arrived at our analytic cohorts. In brief, eligible patients included those initially diagnosed with stage I-IV RCC. Stage I-III patients were included if they had claims for secondary malignant neoplasm on two separate days at any time after their registry recorded initial RCC diagnosis; stage IV patients were included if they had claims-identifiable codes for RCC within 2 months of their registry recorded diagnosis date. The index date was defined as the RCC diagnosis date from the registry for stage IV patients, or the date of the first of the two metastatic diagnoses claims for stage I-III patients.

We defined the modal provider as the individual who was identified most frequently on claims with a diagnosis code of RCC or metastatic cancer between 2 months prior to and 3 months following the index date. Individual providers were identified using their National Provider Identifier code.

We defined two cohorts of patients. The first cohort included all patients with $\mathrm{mRCC}(N=687)$, and our outcome of interest was OAA initiation. For the second cohort, we only included patients with $\mathrm{mRCC}$ from the first cohort who had initiated an OAA and survived the first 90 days post-initiation $(N=207)$. For this cohort, the outcome of interest was OAA adherence. Throughout the manuscript, the first cohort will be referred to as the mRCC cohort and the second cohort will be referred to as the OAA initiator cohort.

\subsection{Study variables}

Oral anticancer agent initiation within the 12 months following the patient's metastatic index date was identified from prescription drug files and pharmacy claims by reviewing generic and brand names as well as national drug codes for the following: sorafenib (2005), sunitinib (2006), pazopanib (2009), everolimus (2009), and axitinib (2012). Adherence to OAAs was defined as having $\geq 80 \%$ proportion of days covered (PDC) for the 90 consecutive days following an initial OAA claim that patients had access to any OAA days' supply. For sunitinib claims, the days' supply was adjusted to account for the " 4 week on, 2 week off" scheduling, to avoid underestimating adherence. ${ }^{7,8,17}$

Provider-level variables included specialty, sex, race/ ethnicity, years in practice, provider's RCC patient volume, and practice locations. Provider specialty was classified as urology/urological surgery, hematology/medical oncology, internal medicine, and other. Provider's years in practice was calculated for each provider-patient encounter as the years elapsed from the provider's medical school graduation to the year of the patient's index date. Provider volume was defined as the frequency of unique RCC patients that the provider treated prior to an $\mathrm{mRCC}$ patient's diagnosis date.

Patient-level control variables of interest included: age at metastatic diagnosis, race/ethnicity, rural location (based on the revised 2010 Rural Urban Commuting Area code categorization ${ }^{18}$ ), patient sex, insurance coverage at metastatic index date, histology, stage at initial diagnosis, radical/partial nephrectomy in the prior year, number of comorbidities at baseline (based on comorbidities included in the Charlson Comorbidity Index ${ }^{19}$ ), the predicted probability of frailty, ${ }^{20}$ and distance to the nearest National Cancer Institute Cancer Center. In addition, patients' area-level socioeconomic context was measured using 2008-2012 American Community Survey 5-year estimates of census tract-level percent with bachelor's degree and percent living below the poverty level.

\subsection{Statistical analysis}

We estimated the frequencies, percentages, medians, and interquartile ranges (IQR) of provider- and patient-level characteristics for both the mRCC and OAA initiator cohorts. Poisson regression with a robust variance estimator was used to estimate the unadjusted and multivariable adjusted risk ratios (RRs) and their corresponding 95\% confidence limits (CLs) to evaluate factors associated with OAA initiation and adherence. As a sensitivity analysis, we used a fractional logit model to evaluate the unadjusted and adjusted PDC.

For both the OAA initiation and adherence analyses, a model reduction method was chosen to account for the analytic sample sizes, the number of study variables of interest, and recommended events per variable (EPV) guidelines. We calculated the maximum allowable number of variables based on at least $10 \mathrm{EPV}$ and used a least absolute shrinkage and selection operator (LASSO) approach to minimize bias in the selection of covariates in the multivariable models from the list of aforementioned provider and patient variables. ${ }^{21}$ Consequently, for both outcomes, all provider-level covariates, including specialty, sex, race/ethnicity, years in practice, RCC patient volume, and practice location were selected for inclusion. When the outcome of interest was OAA initiation, patient-level variables selected for inclusion in multivariate analyses included age, sex, location, insurance, number of comorbid conditions, census tract-level percent with bachelor's degree and percent living below the poverty level, nephrectomy in 12 months prior, stage at diagnosis, distance to the nearest NCI-designated center, and 
Stage I-IV renal cell carcinoma (RCC) diagnosis as first primary diagnosis not identified at autopsy, 2004-2015

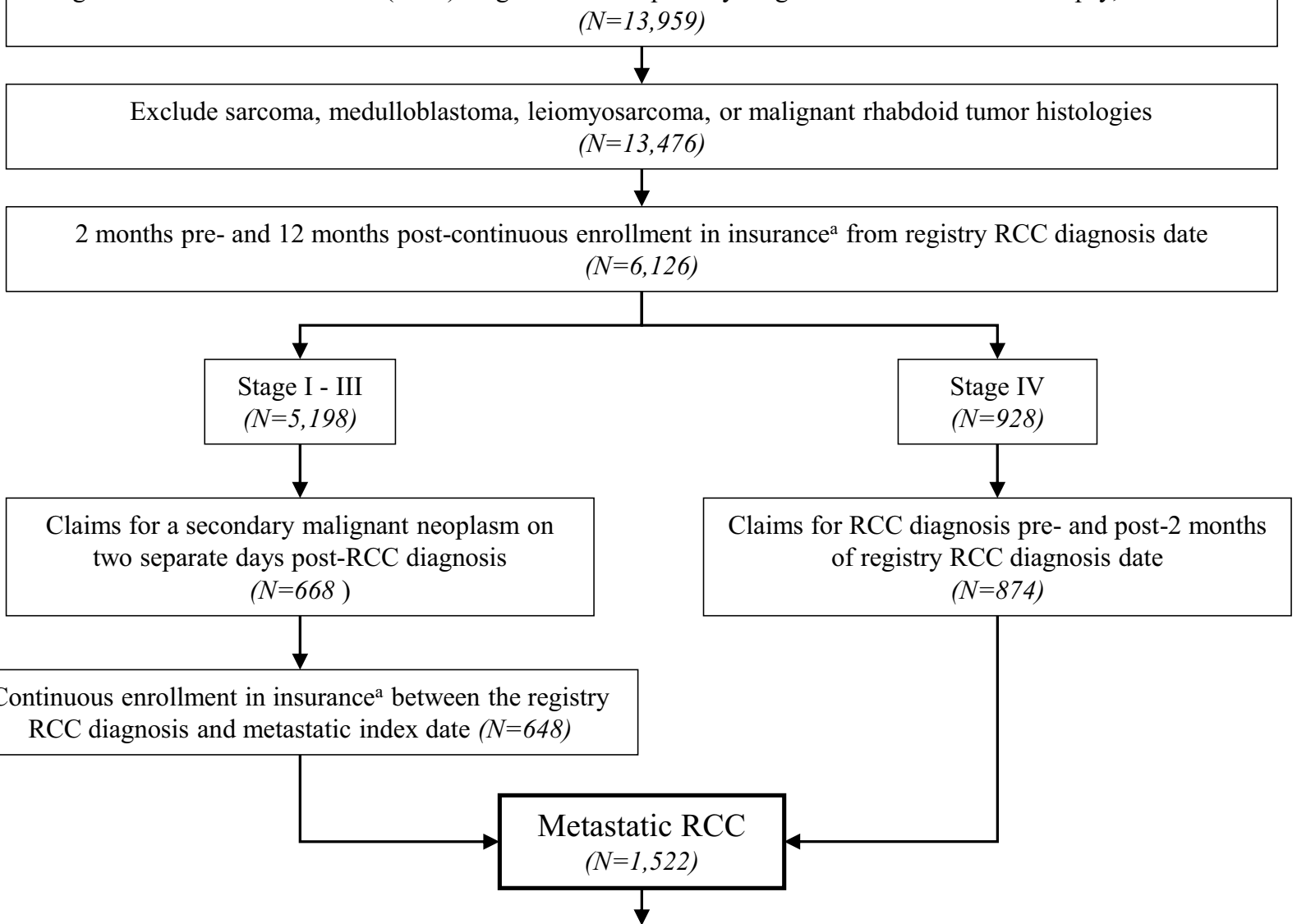

- $>18$ years old on metastatic index date ${ }^{\mathrm{b}}, 2006-2015^{\mathrm{c}}$

$(N=1,293)$

- 12 months of continuous enrollment in insurance pre-metastatic index date ${ }^{a}$

$(N=1,207)$

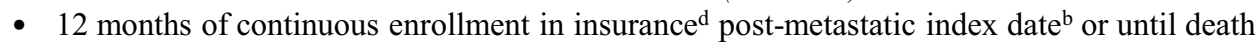
$(N=796)$

- No diagnosis of an additional primary malignancy at a site other than kidney in the 12 months before the metastatic index date ${ }^{b}$

$$
(N=727)
$$

- Excluding patients missing census tract information and whose modal provider could not be identified $(N=713)$

- Excluding patients who could not be linked to an individual modal provider

$$
(N=687)
$$

${ }^{a}$ Required continuous enrollment in Parts A and B for Medicare patients.

${ }^{b}$ Metastatic index date was first metastatic claim date for Stages I-III or first RCC diagnosis date in registry for Stage IV.

${ }^{c}$ Oral anti-cancer agents were first approved for treatment in 2006. Stage I-III patients may have been diagnosed with RCC prior to 2006, but metastatic diagnosis was not till 2006.

d Required continuous enrollment in Parts A, B, and D for Medicare patients. 
frailty. When the outcome of interest was OAA adherence, patient-level variables included in the adjusted model selected using the LASSO approach were age, sex, race/ ethnicity, location, insurance, number of comorbid conditions, census tract-level percent living below the poverty level, nephrectomy in 12 months prior, and distance to the nearest NCI-designated center. Multicollinearity of covariates was assessed using a variance inflation factor of 5 . $p$-values $<0.05$ were considered to be statistically significant. Analyses were conducted using SAS v9.4 (Cary, NC).

\section{3 | RESULTS}

\section{1 | Analytic samples}

Of the 687 patients in the mRCC cohort, 37\% initiated an OAA in the 12 months following metastatic diagnosis. The median age at diagnosis was 70 years, and the majority were male (66\%), NH White (77\%), and urban-residing $(62 \%)$ (Table 1). Most were covered by Medicare only at the time of metastatic diagnosis (63\%). A third of patients had three or more comorbid conditions (33\%). With respect to provider characteristics, most modal providers were with hematologists/medical oncologists (56\%), males (83\%), and $\mathrm{NH}$ white $(74 \%)$, and had a median of 20 years in practice. Compared with the full $\mathrm{mRCC}$ cohort, the initiator cohort $(N=207)$ was younger, had fewer comorbidities, was more often male, and privately insured.

\section{2 | OAA initiation among mRCC patients}

In unadjusted and adjusted models, patients whose modal providers' specialties were other than hematology/medical oncology were far less likely to initiate OAAs compared to patients treated by other specialties. Specifically, in the adjusted models, patients with modal providers specializing in urology/urological surgery $(\mathrm{RR}=0.67 ; 95 \% \mathrm{CI}$ : 0.53 , $0.86)$, internal medicine ( $R R=0.19 ; 95 \% \mathrm{CI}: 0.10,0.36)$, or other specialties ( $R R=0.15$; 95\% CI: $0.05,0.46$ ) were less likely to initiate OAAs compared to patients with modal providers specializing in hematology/medical oncology. In the adjusted model only, patients with female modal providers $(\mathrm{RR}=1.28 ; 95 \% \mathrm{CL}: 1.00,1.62)$ or providers that practiced in only urban areas $(\mathrm{RR}=1.37 ; 95 \% \mathrm{CL}: 1.09$, 1.73) were more likely to initiate OAAs. Additionally, adjusted models indicated that patients ages $80+$ years of age ( $R R=0.39 ; 95 \%$ CL: $0.23,0.66)$ and those with $3+$ comorbid conditions ( $R R=0.72 ; 95 \%$ CL: $0.55,0.94$ ) were less likely to initiate OAAs than, respectively, patients ages 18-49 years and those with no comorbid conditions
(Table 2). Patients with greater predicted frailty were also less likely to initiate OAAs (RR $=0.78$; 95\% CL: 0.63, 0.95).

\section{3 | PDC among OAA initiators}

Among the 207 patients who initiated an OAA and survived at least 90 days after initiation, the median PDC was 0.91 (IQR: $0.66,0.97$ ) (Figure 2). The most frequently prescribed OAAs were sunitinib (57\%) and pazopanib (26\%). Adherence during the initial 90 days was similar for all OAAs except sorafenib (median $=0.79$; IQR: $0.33,0.94$ ), which was lower (Figure 2; Table S1).

Table 3 presents the RRs for the $\geq 80 \%$ PDC adherence outcome. Multivariable analysis showed that those only insured by Medicare were less likely to be adherent $(\mathrm{RR}=0.61 ; 95 \% \mathrm{CL}: 0.42,0.87)$ than those covered by private insurance. Sensitivity analyses demonstrated the mean PDC among privately insured patients was 0.89 , which was significantly higher when compared to patients with Medicaid ( $\triangle \mathrm{PDC}=-0.13$; 95\% CL: -0.22 , $-0.04)$ and with Medicare only $(\triangle \mathrm{PDC}=-0.14 ; 95 \% \mathrm{CL}$ : $-0.20,-0.08$; Table S2).

\section{DISCUSSION}

In this novel study, among patients with mRCC, OAA initiation was significantly associated with provider-level factors, including specialization, sex, and practice location, after controlling for patient-level factors previously shown to be meaningful predictors of OAA initiation in this patient population. ${ }^{6-10}$ Among those who initiated OAAs and survived at least 90 days post-initiation, median PDC was high at 91\%, suggesting substantial overall adherence among diverse patients with $\mathrm{mRCC}$ starting oral therapies. However, publicly insured patients, particularly those on Medicare, have lower adherence to OAAs over time, relative to privately insured patients. In contrast to OAA initiation, provider-level variables were not associated with OAA adherence. Taken together, these findings highlight different barriers to the initiation and maintenance of OAAs.

Several provider-level variables were positively associated with OAA initiation, including having a modal provider in a hematology/oncology specialty. Compared to other specialists, hematologists/oncologists may be better able to address some of the key barriers to initiating OAAs and feel more equipped to manage treatment-related toxicities. Their increased familiarity and experience with OAAs may mean they are more comfortable and adept at educating and counseling patients on using OAAs than other providers. Second, the process of obtaining 
T A B LE 1 Descriptive statistics of the analytic cohorts

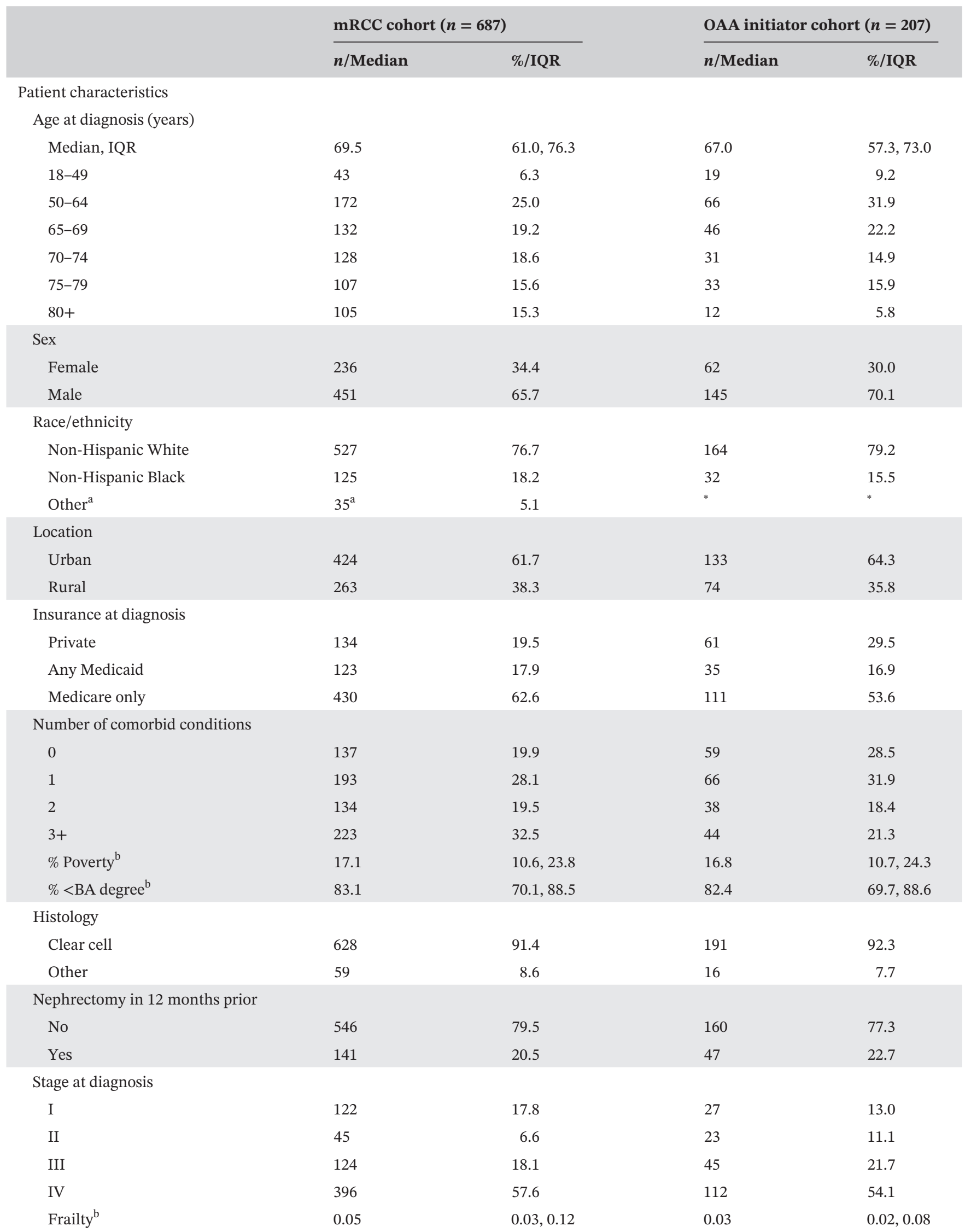

(Continues) 
TABLE 1 (Continued)

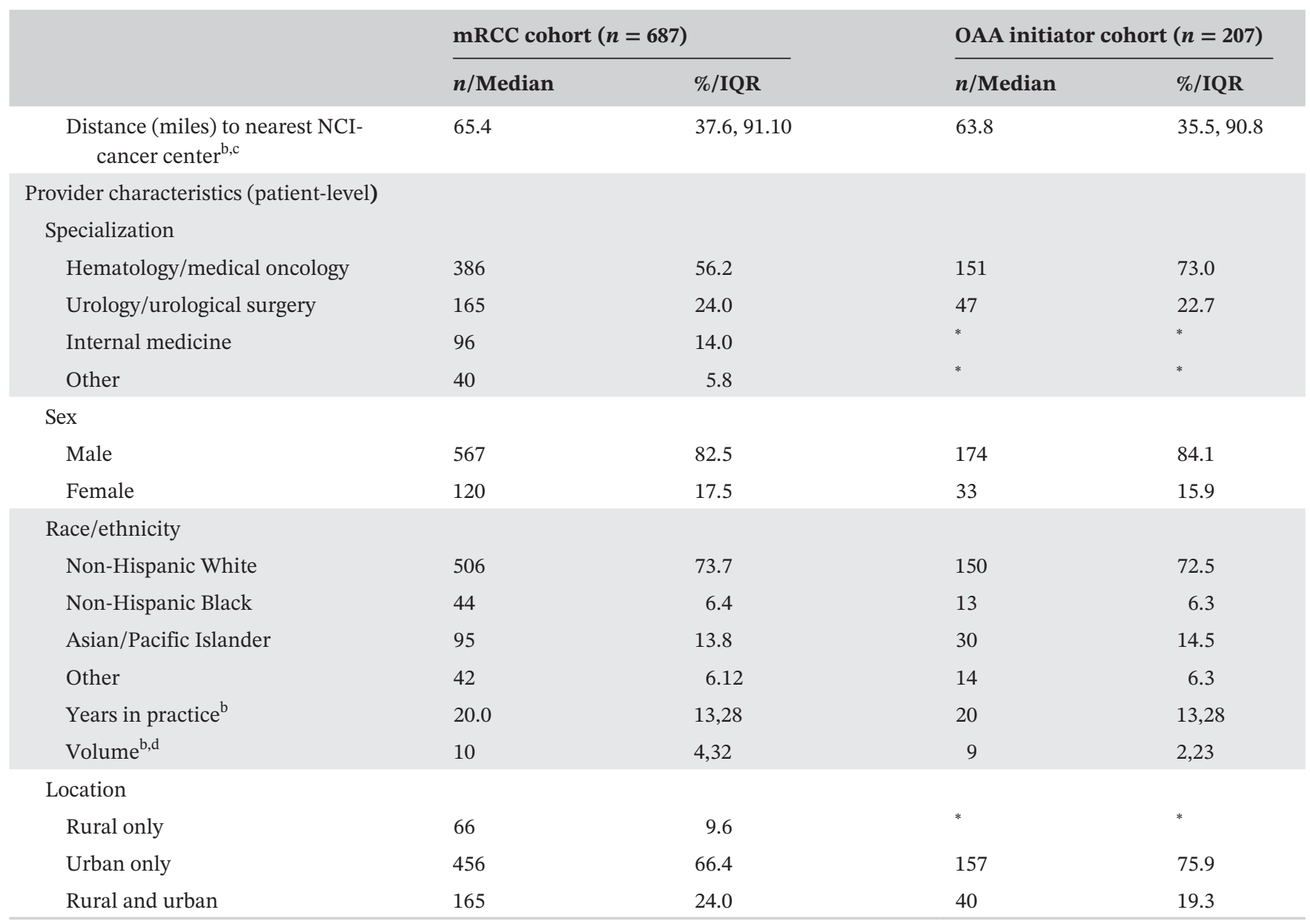

Abbreviations: BA, bachelor; IQR, interquartile range; mRCC, metastatic renal cell carcinoma; NCI, National Cancer Institute; OAA, oral anticancer agent. a“'Other” patient race/ethnicity comprised: non-Hispanic American Indian, non-Hispanic Other, Hispanic White, Hispanic Other, Unknown ethnicity White, and Unknown ethnicity Black.

${ }^{\mathrm{b}}$ Median and IQR are shown.

${ }^{\mathrm{c}} \mathrm{NCI}$ centers were included from states contiguous with North Carolina: Georgia, South Carolina, Tennessee, and Virginia.

${ }^{\mathrm{d}}$ Provider volume was defined as the frequency of unique RCC patients treated prior to a mRCC diagnosis.

*Indicates cell value is $\leq 11$ and is suppressed to protect patients' confidentiality.

insurance authorization of OAAs is onerous and may represent an impediment to prescribing OAAs for providers who are not routinely managing cancer patients. ${ }^{13}$ For example, medical oncology practices are more likely to have clinical oncology pharmacists or other staff specially trained in facilitating access to OAAs. ${ }^{22}$ Not only does this reduce a potential process barrier to accessing OAAs, but strong care coordination through oncology and pharmacy providers has been associated with patients' beliefs in the necessity of OAAs and the perceived benefits of taking OAAs, which may affect the long-term adherence. ${ }^{23}$

Providers practicing in urban areas were also more likely to initiate OAAs. Significant differences in OAA initiation have been documented among cancer patients residing in rural and urban areas. ${ }^{24-26}$ These disparities may be partially attributed to the differences in resources and clinical volume between urban and rural practices. In a previous study among patients with prostate and kidney cancer receiving OAAs, $73 \%$ of all OAA prescriptions required two or more phone calls by clinic staff, and $40 \%$ required five or more calls. ${ }^{27}$ Providers practicing in urban clinics may have more staff they can rely on to ensure that patients' prescriptions are authorized and completed than smaller, rural practices. Furthermore, providers in rural areas are more likely to be professionally isolated and face barriers to continuing medical education, ${ }^{28}$ potentially making them less aware of current guideline and best treatment practices.

Even though OAA adherence was high, PDC was 14 percentage points lower among Medicare patients than those with private insurance, even when normalized for age, frailty, and comorbid conditions. This disparity, in part, may be due to Medicare patients facing significant cost barriers. Compared to privately insured patients, 
TA B L E 2 Risk ratios (RRs) for OAA initiation among patients with mRCC $(N=687)$

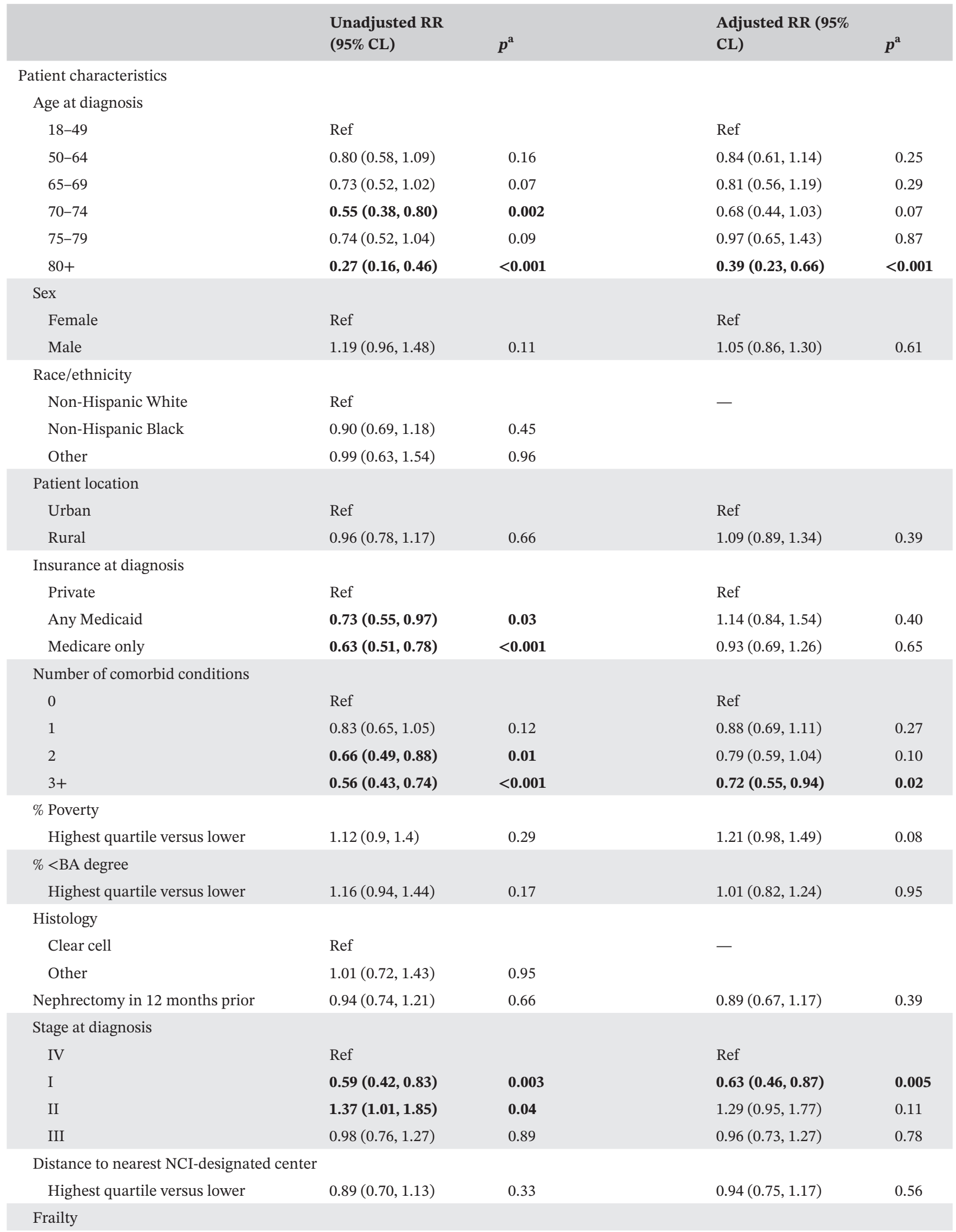


TABLE 2 (Continued)

\begin{tabular}{|c|c|c|c|c|}
\hline & $\begin{array}{l}\text { Unadjusted RR } \\
(95 \% \mathrm{CL})\end{array}$ & $p^{\mathbf{a}}$ & $\begin{array}{l}\text { Adjusted RR (95\% } \\
\text { CL) }\end{array}$ & $p^{\mathbf{a}}$ \\
\hline Above median versus below & $0.64(0.52,0.79)$ & $<0.001$ & $0.78(0.63,0.95)$ & 0.02 \\
\hline \multicolumn{5}{|l|}{ Specialization } \\
\hline Hematology/medical oncology & Ref & & Ref & \\
\hline Internal medicine & $0.19(0.10,0.36)$ & $<0.001$ & $0.22(0.12,0.41)$ & $<0.001$ \\
\hline Other & $0.15(0.05,0.46)$ & 0.0008 & $0.19(0.06,0.55)$ & 0.002 \\
\hline \multicolumn{5}{|l|}{ Sex } \\
\hline Male & Ref & & Ref & \\
\hline Non-Hispanic Black & $1.07(0.72,1.57)$ & 0.75 & $1.32(0.90,1.93)$ & 0.15 \\
\hline Asian/Pacific Islander & $1.07(0.81,1.42)$ & 0.61 & $0.98(0.77,1.24)$ & 0.84 \\
\hline Other & $1.06(0.70,1.60)$ & 0.78 & $1.17(0.78,1.76)$ & 0.45 \\
\hline Years in practice ${ }^{b}$ & $1.00(1.00,1.00)$ & 0.92 & $1.00(1.00,1.00)$ & 0.66 \\
\hline Volume $^{c}$ & $0.99(0.98,1.01)$ & 0.26 & $0.99(0.98,1.01)$ & 0.43 \\
\hline \multicolumn{5}{|l|}{ Location } \\
\hline Rural only & $0.62(0.36,1.06)$ & 0.08 & $0.84(0.49,1.43)$ & 0.52 \\
\hline Urban only & $1.28(1.00,1.64)$ & 0.05 & $1.37(1.09,1.73)$ & 0.007 \\
\hline Rural and urban & Ref & & Ref & \\
\hline
\end{tabular}

Abbreviations: BA, bachelor; CL, confidence limits; mRCC, metastatic renal cell carcinoma; NCI, National Cancer Institute; OAA, oral anticancer agent.

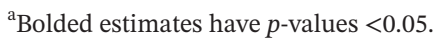

${ }^{\mathrm{b}}$ Years in practice was scaled to 5 years.

${ }^{\mathrm{c}}$ Provider volume was scaled to four RCC patients.

FIG URE 2 Distribution of proportion of days covered from first prescribed oral anticancer agent (OAA) over 90 days following initial OAA claim, stratified by select OAAs. The circles represent the mean and heavy vertical line represents the median. The box represents the interquartile range and whiskers represent the range of values. The category "Other" includes axitinib and everolimus. **indicates cell value is $\leq 11$ and is suppressed to protect patients' confidentiality

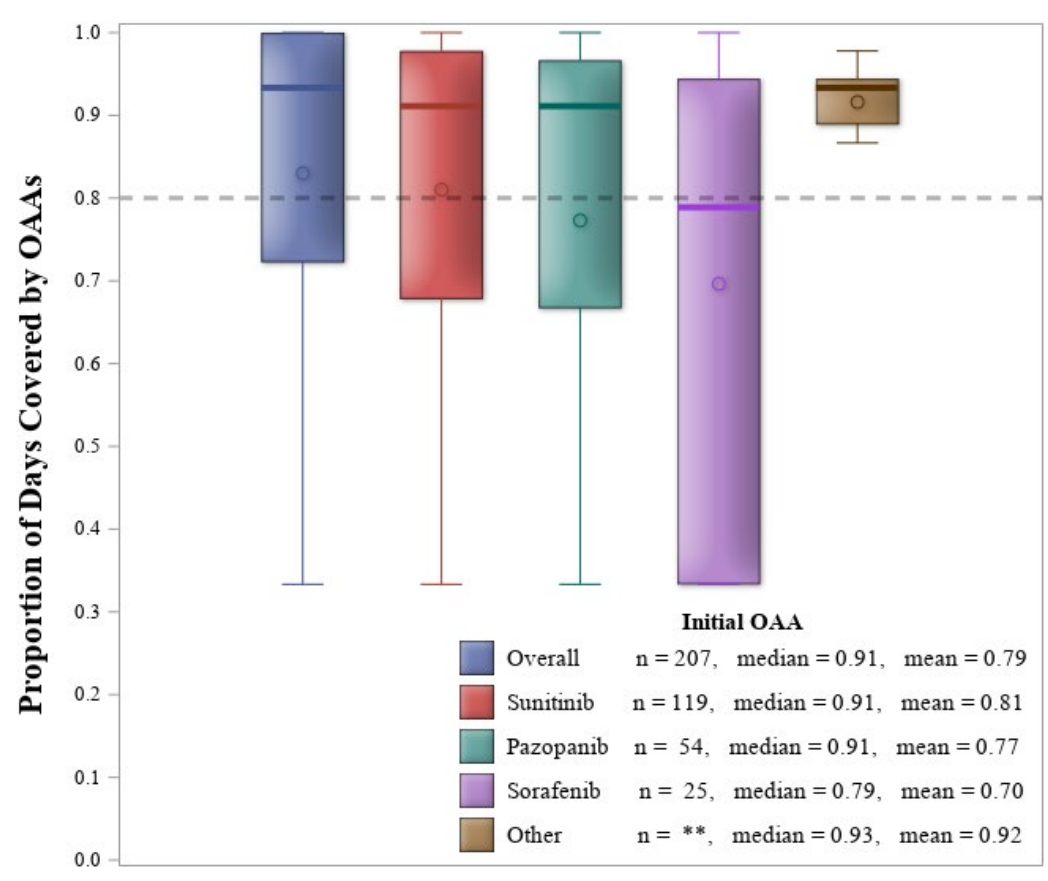


T A B L E 3 Risk ratios (RRs) for 80\% OAA adherent among patients with mRCC who survived at least 90 days post-OAA initiation $(N=207)$

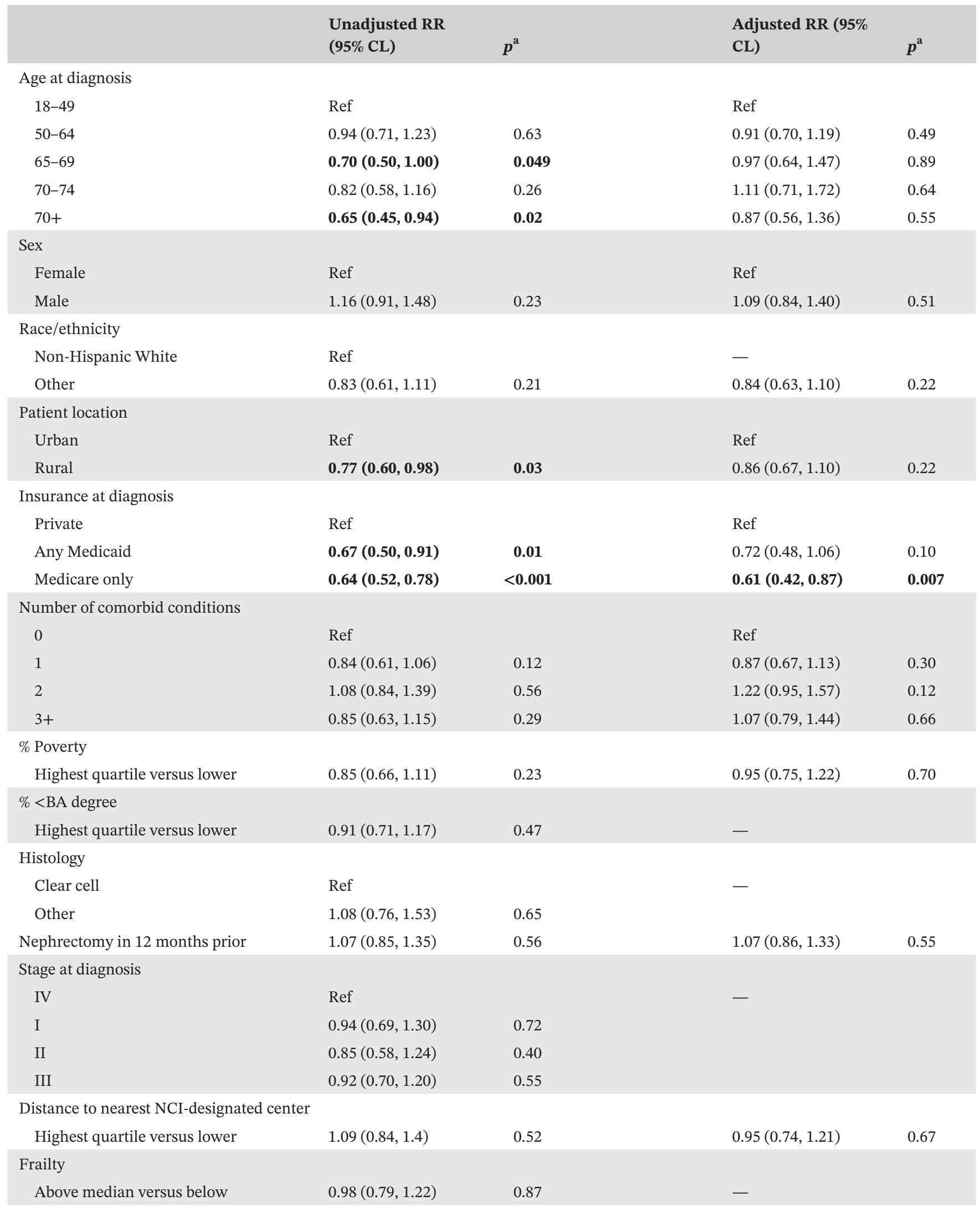

Provider characteristics 
TABLE 3 (Continued)

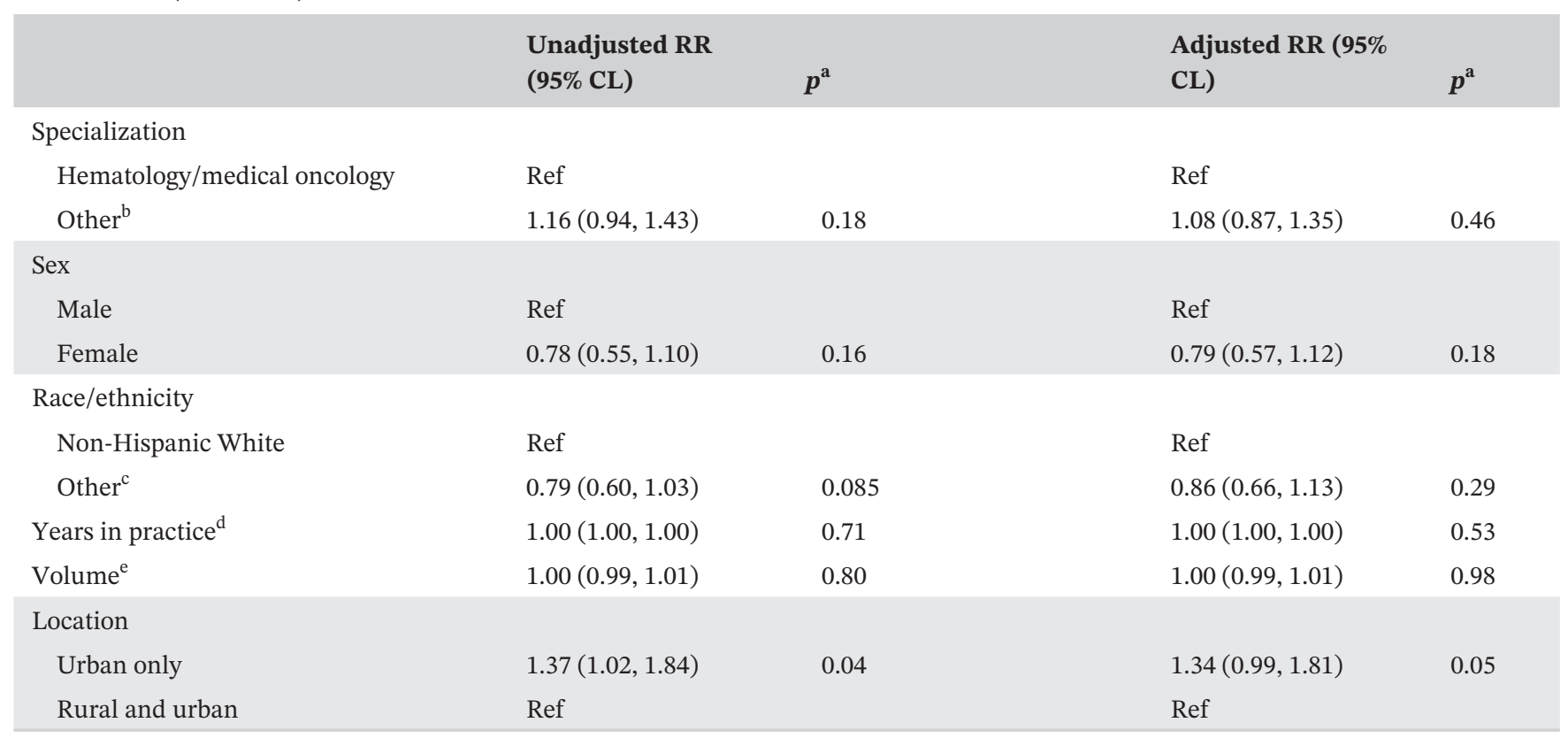

Abbreviations: BA, bachelor; CL, confidence limits; mRCC, metastatic renal cell carcinoma; OAA, oral anti-cancer agent.

${ }^{\mathrm{a} B o l d e d}$ estimates have $p$-values $<0.05$.

${ }^{b}$ Due to small cell sizes, categories of urology/urological surgery, internal medicine, and other were collapsed into one category.

${ }^{\mathrm{c}}$ Due to small cell sizes, catergories of Non-Hispanic Black, Asian/Pacific Islander, and other were collapsed into one category.

${ }^{\mathrm{d}}$ Years in practice was scaled to 5 years.

${ }^{\text {e}}$ Provider volume was scaled to 4 RCC patients and covers the period between the patient's metastatic index date and all prior years of data.

Medicare patients generally have higher coinsurance costs for specialty drugs, and there is no cap on out-ofpocket spending for outpatient prescription drugs. ${ }^{13,29}$ Consequently, as out-of-pocket spending from OAAs accumulates, the financial burden experienced by Medicare patients may reduce their OAA adherence over time. While $78 \%$ of OAA prescriptions filled with Medicare Part $D$ receive some type of charitable assistance, these programs often only cover about $15 \%$ of the OAA prescription's cash price. ${ }^{30}$ If OAA prices continue to annually increase by $12 \%,{ }^{31}$ these insurance disparities may further harm OAA adherence and potentially also influence OAA initiation, particularly in Medicare-insured individuals.

There are limitations of this study that should be noted. First, our study only includes individuals from a single state, potentially limiting the generalizability of our findings. However, the present study from a racially, geographically, and socioeconomically diverse cohort confirms and reinforces findings from other claims-based studies using MarketScan and SEER-Medicare, showing that age and comorbidities are associated with OAA initiation. ${ }^{9,32}$ Importantly, our study adds provider-level predictors to the literature on mRCC OAA use. Second, while we were able to observe the provider visits for which a patient had insurance claims to identify the modal provider, we do not know the content of those visits and assumed, as other analyses have been performed, that the modal provider was most likely to have influence over patients' OAA use. Lastly, OAA adherence was measured using filled prescription records rather than observing actual medication use; filled prescriptions do not necessarily indicate they were consumed, but this is a common approach in the literature that is used to understand medication use. ${ }^{6,8}$

Using a population-based, multi-payer sample, our results suggest that provider characteristics, including specialty and location, are linked with OAA initiation, although more information is needed to better understand provider decision-making, knowledge, and level of comfort prescribing and managing OAAs in this patient population. Finally, while OAA adherence was high overall, we found evidence of disparities in use among Medicare patients compared to privately insured patients. Future research should examine the role that insurance plays within the larger healthcare context and particularly how it interacts with OAA adherence over time. ${ }^{13}$

\section{ETHICS STATEMENT}

This study received exemption status by the University of North Carolina Institutional Review Board (\#19-0451).

\section{CONFLICTS OF INTEREST}

LPS and BEJ receive unrelated funding paid to their institution from AstraZeneca. SBW has received unrelated grant funding paid to her institution from Pfizer Foundation/ 
NCCN and AstraZeneca. TZ has research funding and consulting relationships with Pfizer, Merck, and Novartis, and consulting relationships with Exelixis, Calithera, and Bayer. DG has a current relationship with Bayer, Novartis, Merck \& Co., Exelixis, and Pfizer. CG has received research funding from the NCCN Foundation, Genentech, Johnson \& Johnson, and funding from Flatiron Inc. for travel to and speaking at a scientific conference. CS has research funding with Pfizer, Exelixis, Merck, and BMS.

\section{DATA AVAILABILITY STATEMENT}

The individual registry and claims data (even de-identified) used and/or analyzed during this study are not publicly available due to the Cancer Information and Population Health Resource (CIPHR) policies. Collaboration requests and data use agreements with CIPHR (https://ciphr.unc. edu/) are necessary to obtain access to the de-identified data.

\section{ORCID}

Lisa P. Spees (1) https://orcid.org/0000-0002-5680-9330

Tian Zhang (1) https://orcid.org/0000-0001-8914-3531

Cary P. Gross (D) https://orcid.org/0000-0002-4974-935X

\section{REFERENCES}

1. Siegel RL, Miller KD, Jemal A. Cancer statistics, 2016. CA Cancer J Clin. 2016;66(1):7-30.

2. Kidney and Renal Pelvis Cancer-Cancer Stat Facts [Internet]. [cited 2021 Feb 15]. https://seer.cancer.gov/statfacts/html/ kidrp.html

3. Shih YCT, Xu Y, Chien CR, et al. Rising economic burden of renal cell carcinoma among elderly patients in the USA: part II-an updated analysis of SEER-Medicare data. Pharmacoeconomics. 2019;37(12):1495-1507.

4. Weingart SN, Brown E, Bach PB, et al. NCCN Task Force Report: oral chemotherapy. J Natl Compr Cancer Netw. 2008;6(S3):S-1-S-14.

5. Banna GL, Collovà E, Gebbia V, et al. Anticancer oral therapy: emerging related issues. Cancer Treat Rev. 2010;36:595-605.

6. Miller LA, Stemkowski S, Saverno K, et al. Patterns of care in patients with metastatic renal cell carcinoma among a U.S. Payer population with commercial or Medicare Advantage membership. J Manag Care Spec Pharm. 2016;22(3):219-226.

7. Vogelzang NJ, Pal SK, Ghate SR, et al. Real-world economic outcomes during time on treatment among patients who initiated sunitinib or pazopanib as first targeted therapy for advanced renal cell carcinoma: a retrospective analysis of medicare claims data. J Manag Care Spec Pharm. 2018;24(6):525-533a.

8. Racsa PN, Whisman TR, Worley K. Comparing two tyrosine kinase inhibitors for treatment of advanced renal cell carcinoma in Medicare and commercially insured patients. Curr Med Res Opin. 2015;31(10):1933-1940.

9. Pal S, Gong J, Mhatre SK, et al. Real-world treatment patterns and adverse events in metastatic renal cell carcinoma from a large US claims database. BMC Cancer. 2019;19(1):548.
10. Wheeler SB, Spees LP, Jackson BE, et al. Patterns and predictors of oral anticancer agent use in diverse patients with metastatic renal cell carcinoma. J Oncol Pract. 2021;in press.

11. Partridge AH. Adherence to therapy with oral antineoplastic agents. J Natl Cancer Inst. 2002;94(9):652-661.

12. Verbrugghe M, Verhaeghe S, Lauwaert K, Beeckman D, Van Hecke A. Determinants and associated factors influencing medication adherence and persistence to oral anticancer drugs: A systematic review. Cancer Treat Rev. 2013;39:610-621.

13. Dean LT, George M, Lee KT, Ashing K. Why individuallevel interventions are not enough: systems-level determinants of oral anticancer medication adherence. Cancer. 2020;126(16):3606-3612.

14. Taplin SH, Price RA, Edwards HM, et al. Introduction: understanding and influencing multilevel factors across the cancer care continuum. J Natl Cancer Inst - Monogr. 2012;2012(44):2-10.

15. Zapka J, Taplin SH, Ganz P, Grunfeld E, Sterba K. Multilevel factors affecting quality: examples from the cancer care continuum. J Natl Cancer Inst - Monogr. 2012;2012(44):11-19.

16. Meyer AM, Olshan AF, Green L, et al. Big data for populationbased cancer research: the integrated cancer information and surveillance system. NC Med J. 2014;75(4):265-269.

17. MacLean E, Mardekian J, Cisar LA, Hoang CJ, Harnett J. Realworld treatment patterns and costs for patients with renal cell carcinoma initiating treatment with sunitinib and pazopanib. $J$ Manag Care Spec Pharm. 2016;22(8):979-990.

18. USDA ERS - Rural-Urban Commuting Area Codes [Internet]. [cited 2021 Mar 23]. https://www.ers.usda.gov/data-products/ rural-urban-commuting-area-codes.aspx

19. Charlson ME, Pompei P, Ales KL, MacKenzie CR. A new method of classifying prognostic comorbidity in longitudinal studies: development and validation. J Chronic Dis. 1987;40(5):373-383.

20. Faurot KR, Jonsson Funk M, Pate V, et al. Using claims data to predict dependency in activities of daily living as a proxy for frailty. Pharmacoepidemiol Drug Saf. 2015;24(1):59-66.

21. Harrell FE, Lee KL, Mark DB. Multivariable prognostic models: issues in developing models, evaluating assumptions and adequacy, and measuring and reducing errors. Stat Med. 1996;15(4):361-387.

22. Muluneh B. Financial toxicity in patients with cancer: what a tangled web we weave. J Nat Compr Cancer Netw. 2021;19:3-5.

23. Cohen Castel O, Shadmi E, Keinan-Boker L, Granot T, Karkabi K, Dagan E. The association between patients' perceived continuity of care and beliefs about oral anticancer treatment. Support Care Cancer. 2019;27(9):3545-3553.

24. Yen TWF, Czypinski LK, Sparapani RA, et al. Socioeconomic factors associated with adjuvant hormone therapy use in older breast cancer survivors. Cancer. 2011;117(2):398-405.

25. Pezzin LE, O’Niel MB, Nattinger AB. The economic consequences of breast cancer adjuvant hormonal treatments. J Gen Intern Med. 2009;24(S2):446-450.

26. Charlson JA, McGinley EL, Nattinger AB, Neuner JM, Pezzin LE. Geographic variation of adjuvant breast cancer therapy initiation in the United States: lessons from medicare Part D. $J$ Natl Compr Cancer Netw. 2017;15(12):1509-1517.

27. Geynisman DM, Meeker CR, Doyle JL, et al. Provider and patient burdens of obtaining oral anticancer medications. Am J Manag Care. 2018;24(4):e128-e133.

28. Jukkala AM, Henly SJ, Lindeke LL. Rural perceptions of continuing professional education. J Contin Educ Nurs. 2008;39(12):555-563. 
29. Dusetzina SB, Keating NL. Mind the gap: why closing the doughnut hole is insufficient for increasing medicare beneficiary access to oral chemotherapy. J Clin Oncol. 2016;34(4):375-380.

30. Zullig LL, Wolf S, Vlastelica L, Shankaran V, Yousuf ZS. The role of patient financial assistance programs in reducing costs for cancer patients. J Manag Care Spec Pharm. 2017;23(4):407-411.

31. Shih YCT, Xu Y, Liu L, Smieliauskas F. Rising prices of targeted oral anticancer medications and associated financial burden on medicare beneficiaries. J Clin Oncol. 2017;35(22):2482-2489.

32. Wilson L, Spees L, Pritchard J, et al. Real-world use of oral anticancer agents and related costs in elderly patients with metastatic renal cell carcinoma in the United States. In ASCO Quality Care Symposium. 2020.

\section{SUPPORTING INFORMATION}

Additional supporting information may be found online in the Supporting Information section.

How to cite this article: Spees LP, Wheeler SB, Jackson BE, et al. Provider- and patient-level predictors of oral anticancer agent initiation and adherence in patients with metastatic renal cell carcinoma. Cancer Med. 2021;10:6653-6665. https:// doi.org/10.1002/cam4.4201 